\title{
COHERENT FUSION OF INFORMATION FOR OPTIMAL DETECTION IN SENSOR NETWORKS
}

\author{
David Ramírez, Javier Vía and Ignacio Santamaría \\ Communications Engineering Dept., University of Cantabria, Santander, 39005, Spain. \\ e-mail: \{ramirezgd, jvia, nacho\}@gtas.dicom.unican.es
}

\begin{abstract}
In this work, we consider the problem of centralized detection in wireless sensor networks when the sensors transmit coherently through a multiple access channel. We derive the optimal weighting at each sensor that maximizes the error exponent. Firstly, the noiseless case is considered and a closedform solution to the problem is found. Secondly, we generalize the formulation to consider additive noise at the fusion center. For the noisy case, we propose a suboptimal approach which allows us to find a closed-form solution. Interestingly, the proposed approaches reduce to the extraction of a normalized eigenvector of a generalized eigenvalue problem. The performance of the proposed scheme is illustrated by means of numerical results, showing that the suboptimal approach has a similar performance to that of the optimal one; and that the proposed scheme outperforms other techniques, such as orthogonal transmissions or the maximization of the signalto-noise ratio.
\end{abstract}

Index Terms - Wireless sensor networks (WSNs), error exponent, centralized detection, Kullback-Leibler divergence, multiple access channel.

\section{INTRODUCTION}

In the last years, wireless sensor networks (WSNs) have emerged as a key technology to collect geographically distributed data due to their easy deployment. In WSN, each sensor could have some intelligence or only transmit the measurements to the fusion center (FC), which has to process this information according to some task such as detection or estimation. In this work, we focus on the problem of centralized detection, where the FC has to decide between two different models for the observed signal. In particular, we consider the data transmission through a multiple access channel (MAC), and therefore, there are two possibilities: orthogonal transmission and coherent transmission [1].

This work was supported by the Spanish Government, Ministerio de Ciencia e Innovación (MICINN), under projects MultiMIMO (TEC200768020-C04-02) and COMONSENS (CSD2008-00010, CONSOLIDERINGENIO 2010 Program) and FPU grant AP2006-2965.
When the information is transmitted coherently, some type of processing is needed at the sensors in order to improve the performance of the detector. Specifically, we propose to use a sensor processing which consists of a simple complex weighting. The optimal weights are found by maximizing the error exponent of the miss probability [2], which is easily found. In fact, when the samples are independent and identically distributed (i.i.d), the Stein's Lemma states that the error exponent is given by the Kullback-Leibler divergence between the probability density functions of both hypotheses [2].

In this paper, we first consider the noise-free case, and show that the solution to this problem is given by the eigenvector of a generalized eigenvalue problem. Secondly, we consider additive noise at the FC which results into a complicated non-convex optimization problem. However, we present a suboptimal approach, which regularizes the correlation matrices under both hypotheses and allows us to obtain a closedform solution. Finally, numerical examples are presented to illustrate the performance of the proposed technique. These results show that the coherent approach based on the maximization of the error exponent presents better performance than other alternative schemes such as orthogonal transmissions or the maximization of the signal-to-noise ratio (SNR).

\section{PROBLEM STATEMENT}

In this work, we consider the problem of centralized detection in a WSN, where each sensor acquires a signal generated by a physical phenomenon, transmits this information to the FC using a multiple access channel (MAC), where a decision based on the information provided by the sensors is taken. This problem can be formulated as follows: given a set of available samples at the FC, it has to decide which of the hypotheses $\mathcal{H}_{1}$ or $\mathcal{H}_{0}$ is true. In particular, the signals acquired by the sensors under both hypotheses, are given by

$$
\begin{aligned}
\mathcal{H}_{1}: & \mathbf{s}[n] \sim \mathcal{C N}\left(\mathbf{0}, \mathbf{R}_{1}\right), n=0, \ldots, N-1 \\
\mathcal{H}_{0}: & \mathbf{s}[n] \sim \mathcal{C N}\left(\mathbf{0}, \mathbf{R}_{0}\right), n=0, \ldots, N-1
\end{aligned}
$$

where $\mathbf{s}[n]=\left[s_{1}[n], \ldots, s_{M}[n]\right]^{T}$ are the measurements of all sensors at time $n, \mathcal{C N}$ represents a circular complex Gaussian random vector, and $\mathbf{R}_{l}$ is the correlation matrix of the 
measurements under the hypothesis $l$. Finally, the sensor measurements $(\mathbf{s}[n])$ are transmitted to the fusion center through a MAC channel, using orthogonal or coherent transmission.

\subsection{Orthogonal transmission}

In this case, the sensors has to spend $M$ times more resources than transmitting coherently. The received signals are given by ${ }^{1}$

$$
\mathbf{y}[n]=\mathbf{s}[n]+\mathbf{r}[n],
$$

where $\mathbf{r}[n] \sim \mathcal{C N}\left(0, \sigma_{F C}^{2} \mathbf{I}\right)$ is the noise at the $\mathrm{FC}$ and the hypotheses testing is the following

$$
\mathcal{H}_{l}: \quad \mathbf{y}[n] \sim \mathcal{C N}\left(\mathbf{0}, \tilde{\mathbf{R}}_{l}\right), n=0, \ldots, N-1
$$

where $\tilde{\mathbf{R}}_{l}$ are the following matrices

$$
\tilde{\mathbf{R}}_{l}=\mathbf{R}_{l}+\sigma_{F C}^{2} \mathbf{I}, \quad l=0,1,
$$

and $\mathbf{I}$ is the identity matrix. Finally, assuming that the correlation matrices, $\mathbf{R}_{1}$ and $\mathbf{R}_{0}$, are known at the FC (or previously estimated), the optimal test is given by

$$
\operatorname{Tr}\left[\left(\tilde{\mathbf{R}}_{0}^{-1}-\tilde{\mathbf{R}}_{1}^{-1}\right) \hat{\mathbf{R}}\right] \underset{\mathcal{H}_{0}}{\stackrel{\mathcal{H}_{1}}{\gtrless}} \nu_{o},
$$

where $\nu_{o}$ is a properly selected threshold, $\operatorname{Tr}(\cdot)$ denotes the trace of a matrix and

$$
\hat{\mathbf{R}}=\frac{1}{N} \sum_{n=0}^{N-1} \mathbf{y}[n] \mathbf{y}^{H}[n],
$$

can be seen as the estimated correlation matrix.

\subsection{Coherent transmission}

The second option consists of the coherent transmission of all sensor observations. Contrary to the orthogonal case, we only spend $L=N$ time slots to transmit all measurements. We have considered a linear processing previous to the transmission, in which the signal transmitted by the $k$-th sensor is given by $w_{k}^{*} s_{k}[n]$. The received signal at the fusion center is

$$
y[n]=\mathbf{w}^{H} \mathbf{s}[n]+r[n],
$$

where $\mathbf{w}=\left[w_{1}, \ldots, w_{M}\right]^{T}, r[n] \sim \mathcal{C N}\left(0, \sigma_{F C}^{2}\right)$. Thus, the hypotheses are

$$
\mathcal{H}_{l}: \quad y[n] \sim \mathcal{C N}\left(0, \sigma_{l}^{2}\right), n=0, \ldots, N-1
$$

where $\sigma_{l}^{2}=\mathbf{w}^{H} \mathbf{R}_{l} \mathbf{w}+\sigma_{F C}^{2}$ and the optimal test is given by [3, pag. 143]

$$
\left(\sigma_{1}^{2}-\sigma_{0}^{2}\right) \sum_{n=0}^{N-1}|y[n]|^{2} \underset{\mathcal{H}_{0}}{\stackrel{\mathcal{H}_{1}}{\gtrless}} \nu_{c} .
$$

\footnotetext{
${ }^{1}$ Although we have not considered the channel, its effects can be taken into account by modifying the correlation matrices.
}

\subsection{Error exponent}

In this subsection, we present a brief review of the error exponent [2]. The error exponent for the miss probability ${ }^{2}$, assuming a fixed false alarm probability, is defined as

$$
K_{M}=\lim _{N \rightarrow \infty}-\frac{1}{N} \log p_{M}
$$

where $p_{M}$ is the miss probability. This measure is important because it gives an idea of the asymptotic behavior of the detector and, as we will show, it is easier to obtain than the actual miss probability. In fact, the Stein's Lemma [2] provides a simple closed form for the error exponent. Given $N$ samples drawn i.i.d., the error exponent of the miss probability, assuming a Neyman-Pearson detector, is given by

$$
K_{M}=D\left(p_{0} \| p_{1}\right)
$$

where $D\left(p_{0}|| p_{1}\right)$ denotes the Kullback-Leibler divergence between the densities $p_{0}$ and $p_{1}$, which in the case of two multivariate zero-mean Gaussian random variables reduces to

$$
\begin{aligned}
& D\left(\mathcal{C N}\left(0, \mathbf{R}_{0}\right) \| \mathcal{C N}\left(0, \mathbf{R}_{1}\right)\right)= \\
& =\log \left[\frac{\operatorname{det}\left(\mathbf{R}_{1}\right)}{\operatorname{det}\left(\mathbf{R}_{0}\right)}\right]+\operatorname{Tr}\left(\mathbf{R}_{1}^{-1} \mathbf{R}_{0}\right)-M .
\end{aligned}
$$

Finally, we must point out that the use of asymptotic measures have also been proposed by other authors. For instance, in [4], the problem of signal selection in radar and communications is solved using the J-divergence and the Bhattacharyya distance.

\section{MAXIMIZATION OF THE ERROR EXPONENT}

In this section, we design the optimal weight for each sensor based on the maximization of the error exponent. Firstly, we present the solution to the noise-free scenario and, secondly, the problem of noisy links is presented.

\subsection{Noise-free case}

In the noiseless case, the pdfs of the received signal under both hypotheses, assuming a coherent transmission, are given by (2) when $\sigma_{F C}^{2}=0$. The goal is to maximize the KL divergence of these univariate pdfs under the following power constraint

$$
\max _{l=0,1} \mathbf{w}^{H} \mathbf{D}_{l} \mathbf{w} \leq P
$$

where $P$ is the power and $\mathbf{D}_{l}=\operatorname{diag}\left(\mathbf{R}_{l}\right)$ is a diagonal matrix obtained from the main diagonal of $\mathbf{R}_{l}$. Then, the problem to

\footnotetext{
${ }^{2}$ There are other error exponents, for instance, of the false alarm probability or the error probability. However, for the sake of space, we only consider the error exponent of the miss probability.
} 
solve is

$$
\begin{array}{ll}
\underset{\mathbf{w}}{\operatorname{maximize}} & \log \frac{\mathbf{w}^{H} \mathbf{R}_{1} \mathbf{w}}{\mathbf{w}^{H} \mathbf{R}_{0} \mathbf{w}}+\frac{\mathbf{w}^{H} \mathbf{R}_{0} \mathbf{w}}{\mathbf{w}^{H} \mathbf{R}_{1} \mathbf{w}}-1, \\
\text { subject to } & \mathbf{w}^{H} \mathbf{D}_{1} \mathbf{w} \leq P, \\
& \mathbf{w}^{H} \mathbf{D}_{0} \mathbf{w} \leq P .
\end{array}
$$

Since the objective function does not depend on $\|\mathbf{w}\|^{2}$, this allow us to solve the problem in two steps. Firstly, we obtain the weights that maximize the error exponent, which are given by the eigenvector of the following generalized eigenvalue problem $(\mathrm{GEV})$

$$
\mathbf{R}_{1} \mathbf{v}=\lambda \mathbf{R}_{0} \mathbf{v}
$$

associated to the eigenvalue, $\lambda$, which maximizes

$$
K_{M}=\log \lambda+\frac{1}{\lambda}-1,
$$

Finally, the eigenvector is normalized as follows to fulfill the power restrictions

$$
\mathbf{w}=\mathbf{v} \sqrt{\frac{P}{\max _{l=0,1} \mathbf{v}^{H} \mathbf{D}_{l} \mathbf{v}}} .
$$

Let us analyze the function in (5), which is shown in Fig. 1. This function presents its minimum at $\lambda=1$, which is obvious since we can not distinguish between two Gaussians with the same mean and variance. Moreover, since the function is monotone at both sides of the minimum, the maximum should be located at one of its extremes, and therefore, it is only necessary to evaluate (5) for the largest and the smallest eigenvalues. It is also important to notice that the slope of $K_{M}$ is much larger for $\lambda<1$ which provokes that small changes in the eigenvalue turn into large changes in the error exponent.

\subsection{Noisy case}

When additive Gaussian noise is considered at the FC, the problem of maximizing the error exponent is

$$
\begin{aligned}
\underset{\mathbf{w}}{\operatorname{maximize}} \log \frac{\mathbf{w}^{H} \mathbf{R}_{1} \mathbf{w}}{\mathbf{w}^{H} \mathbf{R}_{0} \mathbf{w}}+\sigma_{F C}^{2} & \sigma_{F C}^{2} \\
& +\frac{\mathbf{w}^{H} \mathbf{R}_{0} \mathbf{w}+\sigma_{F C}^{2}}{\mathbf{w}^{H} \mathbf{R}_{1} \mathbf{w}+\sigma_{F C}^{2}}-1,
\end{aligned}
$$

subject to (3). To the best of our knowledge, this problem does not have a closed-form solution unless the correlation matrices have a particular structure. For instance, if $\mathbf{R}_{0}=$ $\mathbf{D}_{1}$, a closed-form solution can be found taking into account that

$$
\mathbf{w}^{H} \mathbf{R}_{0} \mathbf{w}=\mathbf{w}^{H} \mathbf{D}_{1} \mathbf{w}=\mathbf{w}^{H} \mathbf{D}_{0} \mathbf{w}=P,
$$

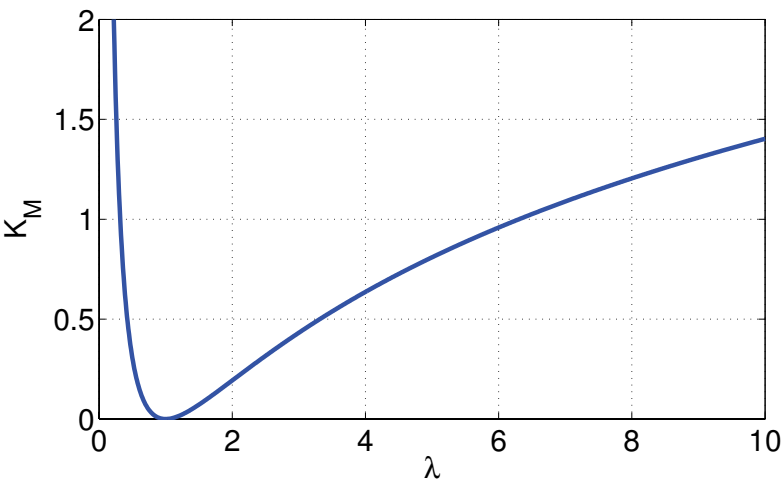

Fig. 1. Error exponent as a function of the eigenvalue of (4) or (6).

and the solution is given by the normalized eigenvector of (4) associated to the eigenvalue which maximizes

$$
K_{M}=\log \frac{\lambda+\sigma_{F C}^{2}}{P+\sigma_{F C}^{2}}+\frac{P+\sigma_{F C}^{2}}{\lambda+\sigma_{F C}^{2}}-1 .
$$

In order to solve the general problem, we propose a suboptimal approach to the optimization problem which allows us to find a closed-form solution instead of using an iterative technique. The approach is based on the regularization of the correlation matrices. By applying this idea, the problem can be rewritten as

$$
\underset{\mathbf{w}}{\operatorname{maximize}} \log \frac{\mathbf{w}^{H} \tilde{\mathbf{R}}_{1} \mathbf{w}}{\mathbf{w}^{H} \tilde{\mathbf{R}}_{0} \mathbf{w}}+\frac{\mathbf{w}^{H} \tilde{\mathbf{R}}_{0} \mathbf{w}}{\mathbf{w}^{H} \tilde{\mathbf{R}}_{1} \mathbf{w}}-1,
$$

subject to (3), where the matrices $\tilde{\mathbf{R}}_{l}$ have been defined in (1). The solution to this problem is similar to that in the noiseless case, i.e., it is given by the normalized eigenvector of the following GEV problem

$$
\tilde{\mathbf{R}}_{1} \mathbf{v}=\lambda \tilde{\mathbf{R}}_{0} \mathbf{v},
$$

associated to the eigenvalue which maximizes (5).

Finally, it is interesting to notice that a similar idea can be found in the field of linear discriminant analysis [5]. However, the approach in [5] does not consider any power restriction, and it is based on the J-divergence, which is a more heuristic measure than the error exponent.

\section{NUMERICAL RESULTS}

In this section, we illustrate the performance of the proposed technique by means of two numerical examples. In both cases, we have considered a sensor network with $M=20$ sensors and the signal-to-noise ratio is $\mathrm{SNR}=P / \sigma_{F C}^{2}=0 \mathrm{dBs}$. In the first example, we have compared the regularized and the 


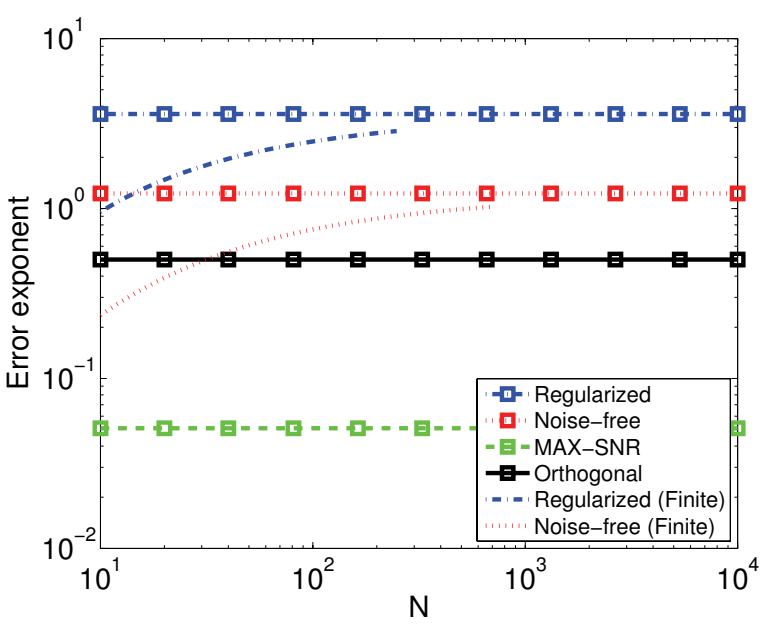

Fig. 2. Error exponent (squares) and finite exponential error rate (unmarked) error exponent of the different techniques.

noise-free approaches presented in Sec. 3, the orthogonal approach and other coherent approach based on the maximization of the SNR (MAX-SNR). The error exponent of the orthogonal approach is given by the KL divergence between the two multivariate Gaussians divided by $M$ since we need $M$ times more slots for transmission and the solution to the maximization of the SNR, assuming equiprobable hypotheses, is given by the normalized eigenvector associated to the largest eigenvalue of the following eigenvalue $(\mathrm{EV})$ problem

$$
\left(\mathbf{R}_{1}+\mathbf{R}_{0}\right) \mathbf{v}=\lambda \mathbf{v}
$$

The error exponents can be seen in Fig. 2 (lines with squares) as well as the finite versions for a false-alarm probability $p_{F A}=0.01$. From this figure, it is obvious that the regularized-based approach is much better than the alternative approaches. Fig. 2 also shows that the error exponent of the orthogonal approach is worse than that of the proposed coherent approaches. On the other hand, under both coherent approaches (regularized and noise-free), the eigenvector associated to the smallest eigenvalue is the one that maximizes the error exponent. Finally, as can be seen, the performance of the MAX-SNR is much worse than the error exponent-based ones, which shows that the error exponent is the right measure to optimize in signal detection.

In the second example, we show that the performance degradation of the suboptimal approach in comparison to the optimal solution is almost negligible. To this end, we use the correlation matrices which allow us to find a closed-form solution $\left(\mathbf{R}_{0}=\mathbf{D}_{1}\right)$. The results of this experiment are shown in Fig. 3, where we can see that the regularized approach provides a performance close to the exact solution.

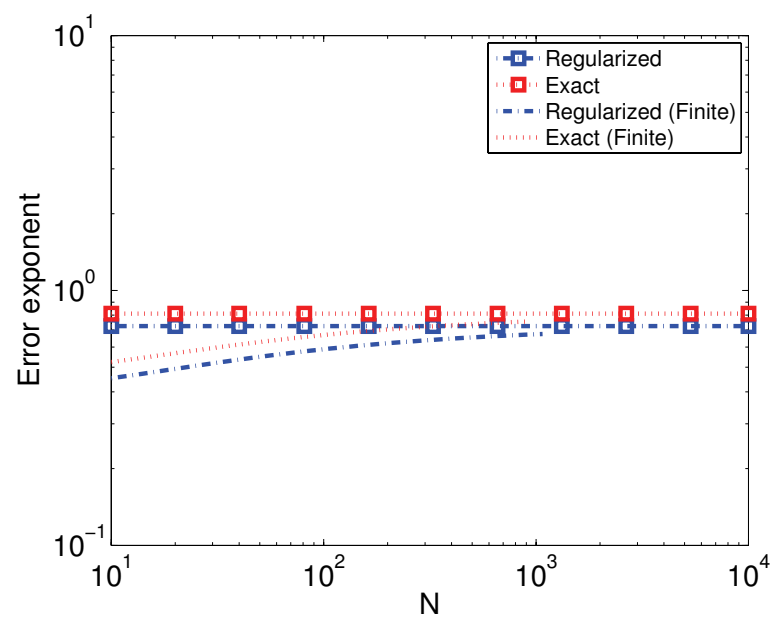

Fig. 3. Comparison of the regularized approach with the optimal solution in a particular example.

\section{CONCLUSIONS}

In this work, we have presented a solution to the problem of centralized detection in sensor networks when the sensors transmit coherently through a multiple access channel. The proposed solution is based on the maximization of the error exponent subject to a network power constraint. In particular, we have obtained the optimal solution for the noise-free case and a closed-form suboptimal solution for the case of noisy links. Finally, the performance of the proposed technique has been illustrated by means of some numerical results, which show that the coherent approach based on the maximization of the error exponent outperforms other techniques, such as orthogonal transmissions or coherent transmissions based on the maximization of the signal-to-noise ratio.

\section{REFERENCES}

[1] Jin-Jun Xiao, Shuguang Cui, Zhi-Quan Luo, and Andrea J. Goldsmith, "Linear coherent decentralized estimation," IEEE Trans. Signal Process., vol. 56, no. 2, pp. 757-770, Feb. 2008.

[2] Thomas M. Cover and Joy A. Thomas, Elements of Information Theory, Wiley-Interscience, 2nd edition, 2006.

[3] S. M. Kay, Fundamentals of Statistical Signal Processing: Detection Theory, vol. II, Prentice Hall, 1998.

[4] T. Kailath, "The Divergence and Bhattacharyya distance measures in signal selection," IEEE Trans. Comm. Tech., vol. 15, no. 1, pp. 52-60, February 1967.

[5] Louis L. Scharf, Statistical Signal Processing: Detection, Estimation, and Time Series Analysis, Addison - Wesley, 1991. 